Voix et Images

volxetimages

\title{
Lettres, fragments et discours amoureux
}

\section{Paul Chanel Malenfant}

Volume 16, numéro 2 (47), hiver 1991

Jovette Marchessault

URI : https://id.erudit.org/iderudit/200908ar

DOI : https://doi.org/10.7202/200908ar

Aller au sommaire du numéro

\section{Éditeur(s)}

Université du Québec à Montréal

\section{ISSN}

0318-9201 (imprimé)

1705-933X (numérique)

Découvrir la revue

\section{Citer cet article}

Malenfant, P. C. (1991). Lettres, fragments et discours amoureux. Voix et Images, 16(2), 345-357. https://doi.org/10.7202/200908ar d'utilisation que vous pouvez consulter en ligne.

https://apropos.erudit.org/fr/usagers/politique-dutilisation/ 


\section{Poésie}

\section{Lettres, fragments et discours amoureux}

\section{par Paul Chanel Malenfant, Université du Québec à Rimouski}

On reconnaît, depuis quelques années et avec la remise en cause des expérimentations de la structure vacante, un « retour du sujet» tant dans telle production romanesque que dans la prolifération biographique et autobiographique, et jusque dans le champ divers des pratiques poétiques. En ce réinvestissement du "je», personnel et singulier, dans le travail des formes et des textes, l'éternel discours amoureux se recycle; il semble parfois s'infléchir de nouveaux affects, comme réactualisé à la faveur de l'angoisse d'une fin de siècle, porté par une conscience alertée du désastre et de la catastrophe. Écrire, dès lors, c'est souvent * écrire l'amour * sous le mode du fragment et de la subjectivité sinistrée, de la discontinuité et de la rupture. L'échec comme la quête amoureuse s'inscrivent dans l'exercice même de l'écriture dont ils constituent à la fois la pulsion et la manière. Écrire est un grand amour, lisait-on à travers le morcellement narratif du Prochain Épisode ${ }^{1}$ d'Hubert Aquin. Et dans la démesure généreuse de Une certaine fin de siècle ${ }^{2}$, Claude Beausoleil proclamait son amour fou de l'écriture. Forme moderne du vertige, résurgence du spleen et de la mélancolie, indice d'une relation revisitée à l'autre que le texte interpelle, le propos d'amour se désigne inévitablement comme mal d'amour, peut-être aussi comme stimulant malaise d'écriture, cela qui la relance, la déplace, l'active en l'énergie de son inachèvement même.

Contrat amoureux et procès d'écriture: voilà donc ce qui se dispose, certes par de bien variables formules, dans nombre des recueils de poèmes portés cette fois à mon attention. Ainsi dans le treizième livre d'Anne-Marie Alonzo, l'Immobile ${ }^{3}$, constitué de douze lettres - lettres d'amour ou grands poèmes épistolaires - adressées à autant de femmes qui ont accompagné le corps à corps de l'auteure avec l'écriture, l'activité scripturale se dit comme "geste", comme présence physique à l'autre. "Faire " le poème, littérảlement, ne se dissocie pas de " faire "l'amour:

J'écris ces jours, j'ecris écoutant ta voix comme repère, j'écris et comme pendant tant de jours d'écriture, ta voix se joint à la mienne, je vois tes yeux, sens tes yeux sur moi, sens ton regard, le sens si près, si fort et lourd, sens ton oil vif, le sens comme 
sentir la pluie à verse. Écrire devient ainsi fiction. Tu n'es plus et pourtant je parle de toi et te parle, à partir de toi, j'écris et t'écris. (p. 78)

Texte de la douleur, discours du corps figé et reclus, ce livre se déploie entre l'oppression de l'inertie et la tension de l'énergie. La phrase cassée, triturée, à l'instar des membres rompus qui l'inscrivent, y alterne avec le développement rythmique, là où l'immobilité aiguise l'imagination du mouvement, là où la syntaxe atteint à l'équilibre diffus de la danse: Entre écrire et écrire ce ballet symbole réel de mouvement puisque danse avant toute choses entre écrire ce ballet se tisse sur les toiles - rêve immobile - trace suivant trace. (p. 72) Équilibre, ainsi se dit tout l'effort de cet amour d'écrire: équilibre entre le pathétique et l'ironie, entre la discrétion et la confidence (cette poésie de la parole), entre le courage et la lamentation, entre le lyrisme contenu et l'effraction sonore du sens. Dans la tension de cet énoncé d'un seul souffle - Aujourd'hui j'écris pour que ma respiration prenne son temps. (p. 41) - comme dans ce laconique constat de l'impossible "pas de deux" - Du ballet à l'hôpital ces mêmes phrases: un, deux ou trois, tenez la jambe, levez le bras. (p. 71) s'exprime la quête de l'harmonie d'un corps perdu, par l'écriture retrouvé.

Du drame personnel d'Anne-Marie Alonzo, tous ses livres antérieurs, depuis Geste ${ }^{4}$ et Veille ${ }^{5}$, entre autres, nous avaient déjà informés; par fragments de récits allusifs, par une écriture elle-même accidentée faisant alterner la bribe autobiographique et la perspective historique, se côtoyer le broyage acoustique des signifiants et la tessiture de l'incantation. L'Immobile nous interdit de nous accoutumer à la tragédie; car ce texte déclame, avec une courageuse solennité où se mêlent les accents bibliques, la difficile sagesse de la solitude amoureuse - et rompue: Alors je préfêre - et ne dis qu'en mon seul nom - vivre loin de tout amour, ne peux supporter d'autre immobilité que la mienne acquise. Que l'âme de l'autre garde son mouvement mais n'entrave plus ce qui peut me rester d'errance. (p. 63). Ainsi, l'Immobile perpétue ce seul mouvement qui emporte tous les autres, le mouvement lent de l'écriture: Elle répéta longuement, le mou-ve-ment, avant de se pencher à nouveau sur ses feuilles pour écrire. (p. 150)

On pourrait trouver comparable éloge de la lenteur, chez Hélène Dorion, dans son recueil au titre magnifique - jaime ces titres qui ouvrent les livres comme des fenêtres -, Un visage appuyé contre le monde ${ }^{6}$. En outre, ce livre commence aussi par une "Lettre" d'amour, comme toutes les lettres... - qui se répercute dans la quatrième partie du texte, "Lettre, encore». Mais tandis que le courrier d'Anne-Marie Alonzo interpellait des destinataires réelles (de Gabrielle Colette à Ludmilla Chiriaeff, de Françoise Loranger à Andrée Lachapelle, entre autres), chez Hélène Dorion, [l]es lettres ne 
franchissent aucune distance (p. 17). C'est dire qu'à l'extrême tangibilité deś corps amoureux présents dans l'Immobile, qu'à la suffocation de l'espace étroit et du temps forclos, s'oppose ici la désolation de l'absence ou de la perte, à défaut d'une emprise sur le réel, sur ce peu d'événements que retient l'univers (p. 26). La fragmentation tient, dans ce discours amoureux, de la dissolution:

Il reste parfois peu de choses: quelques traits sur le visage, les lignes retenues au bout des doigts, des fragments entassés par le temps. Frêles éclats répandus çà et là, comme si la marque légère n'était pas encore la marque, comme si quelques flocons n'étaient pas encore la neige. (p. 11)

Écriture blanche, trace fugitive de ce qui passe sans marque de passage.

Qu'on ne voit pas cependant le seul travail de l'effacement dans cette poésie; on y trouve ce constant effleurement du silence, cet étonnement grave du surgissement de la pensée, comme à l'insu du penseur. Le poème thématise le contour, la surface, le profil ou le seuil. Le champ isotopique de la faille, de la fêlure, de la blessure, de la ruine et du désert, vise moins à traduire le choc ou le fracas que la tranquille usure du monde (p. 45). L'amour se dit dans l'effroi de sa dilution (Aimer nous effraie, chaque fois / frôle en nous la disparition [p. 28]), dans le désastre sans violence de l'habitude: Ce n'est pas la disparition qui étonne, mais cette façon de ne plus l'attendre, alors même qu'on attend encore, parce que toujours la disparition, le manque, sa démesure, - toujours. (p. 32) S'expliquent alors le goût de cette poésie pour le vocable abstrait, son refus de la référence anecdotique et de la nomination prosaïque des objets. Seuls quelques lieux anonymes et de passage (salles d'attente, chambres d'hôtels, halls de gare, corridors: icônes du corps et de sa somnolence...) déterminent la marche et l'écriture ; comme si la terre était une vaste salle de pas perdus, la page, cet espace vacant où se perd le temps périssable. Mais en guise de consolation à la détresse d'exister, le poème se rassérène dans l'émerveillement de l'avancée, la chance de la lettre "mobile»: Lettres - terriblement semblables à nos pas, qui mêlent détresse et apaisement, doute et certitude. (p. 60)

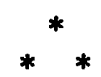

De la faconde, de la volubilité du «beau parleur» aux femmes: le Poèmeroman (p. 9) de Jean Charlebois, Confidentielles ${ }^{7}$, en met plein l'ouie, plein la vue. Ici, l'amour de la langue, le plaisir du jeu de mots, la virtuosité associative, tout concourt à l'euphorique "confusion " du cœur et des "sens". Tout contribue à la profusion d'une écriture débridée, ludique, emportée par le malheur - comme on dit 
* faire un malheur * - ou le bonheur — Qu'en est-il de Don Juan ? de l'expression. Qu'importent alors la joliesse automatique de la tournure (Elles ont les hanches neige avec dedans des dents de lait de louve [p. 11]), la formule qui tient du rébus (Elles a des yeux tout le tour de la bête. Elles prend son mâle en patience [p. 18]), l'inadvertance d'une trop exclusive isotopie culinaire ou nutritive:

Métiers de femme: sel d'entrecuisse, ou sucre d'orge, trous de submersion, poils soyeux insomniaques, pistes d'atterrissage pour lèvres, replis élastiques intimes, bouches. bées, senteur sirop, muscles à modeler, pattes alimentaires, seins faits mains, lèvres louves, jus de fruit. Corps cible. Dans les trous noirs de mes rêves, elles est un nouvel astre ardent. Un autel cinq étoiles. (p. 63)

Car à travers le délire fantasmatique, dans les éclats de voix du discours amoureux, on perçoit, chez Charlebois, l'inscription d'un projet d'écriture dans sa contrapuntique continuité (d'où le remorquage des titres des recueils antérieurs: de Hanches neige ${ }^{8}$ à Corps cible $^{9}$, par exemple); on reconnait, en marge de toutes sectes et comme indifférente à quelque textuelle actualité, la forte personnalité d'une voix, qui par delà la passion du jeu ou du canular, atteint souvent à l'essentiel: Toute cette femme comme un immense délire d'amour amoureux pour que la vie de l'amoureuse et de l'écrivain s'intègre dans la grande vie dont on ne sait rien, qui ne dit rien. (p. 117) Devant l'insoutenable absurdité du monde, le poète, galant jusqu'à la parlure, histoire de plaire, se ravit de la tendre différence des femmes.

A la courtoisie des confidences de Charlebois viennent assez naturellement s'associer les titres («Quelques faits d'armes "; « $\grave{A}$ propos de l'amour courtois "; "Le déclin de la chevalerie»; "D'or, d'azur et de sable») qui ordonnent le recueil d'André Marquis, Nul vieux château ${ }^{10}$. Sur un ton chevaleresque où se confondent Don Quichotte et le preux Roland, s'énoncent des poèmes de capes et d'épées dans une atmosphère de combat, de coups d'éclats et de fracas guerriers. Tout se passe comme s'il s'agissait de renouveler, cette fois dans le cérémonial allégorique de la brutalité et de l'horreur, un langage de la catastrophe moderne et du désastre amoureux:

La tragédie a peu à voir avec le sang bruni des femmes. Dans le désarroi qui est le mien, je revois les batailles, les crânes fendus, les cervelles éclatées. Mais je n'ai plus la force de soulever une épée. Mon bras s'alourdit au poids du jour. Qui suis-je pour m'inquiéter ainsi du sort du monde? (p. 78)

On crée alors l'effet « farouche », celui de la transe et de la panique, de l'élan et de la fièvre, toute constellation établie par le capital sémantique des cataclysmes, fléaux, impostures, infortunes et autres dispositifs du malheur dynamique. 
On pourrait reprocher ici l'emportement de la filature métaphorique ou quelque artifice dans la mise en scène de la toile historique pour accuser le drame de l'incommunicabilité et l'angoisse de l'abolition du monde: le danger éminent un génocide planétaire (p. 25). Cependant, la poésie d'André Marquis atteint ses meilleurs moments - et ce, me semble-t-il, au fur et à mesure de la progression du recueil, en particulier dans les beaux poèmes en prose de la quatrième partie, "D'or, d'azur et de sable * - quand l'affabulation fait place à une conscience attentive à l'Histoire et à la façon dont elle infléchit un destin singulier. Alors, souveraine simplicité du langage, parfaite transparence du propos qui oscille entre optimisme et lucidité:

N'écoute pas ceux qui prétendent que la terre court à sa perte. Depuis la nuit des temps, chaque journée porte en elle la fin du monde. Le vol des oiseaux. L'éclosion des feuilles. La falaise ravinée. Je me disperse un peu plus dans l'azur. Je subis maintes influences. Tout embrasser d'un même regard. Tout soulager d'une seule parole. Mais le vent ne le veut pas ainsi. Une spirale irréversible de la violence. Existe-t-il une seule pierre qui ne soit alphabet, dressée dans l'attente et l'espoir? (p. 79)

Si l'on excepte quelques privautés libidineuses (je m'efforce de te faire languir à la couronne de ton sexe [p. 40]), quelque maniérisme trivial (certaines fouilles mettent à jour une éjaculation aseptisée / dans des draps de senteurs pins épinettes [p. 61]), divers traits de poésie attestent des promesses de cette voix: le retentissement d'une image qui pourrait évoquer le ton - inimitable, pourtant - d'un Paul-Marie Lapointe (napalm essence de menthe / tu te dépouilles de tes voiles / à l'état de promesse dévastatrice" [p. 49]); l'authenticité d'une saillie (nul n'est à l'abri d'une meurtrière [p. 73]); enfin, surtout, la fréquente inscription d'une langue drue, sans la censure civilisée, toute adonnée à l'ardeur du désir, et comme proférée dans la ferveur d'un monde redevenu primitif.

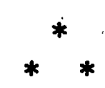

Jexprimais le regret, dans ma première chronique à Voix et images, que nombre des récipiendaires du prix Octave-Crémazie soient restés confinés à la tentative sans suite du premier livrè. Je me ravis de ma ponctuelle méprise, car avec Où serons-nous dans une heure ${ }^{11}$, Jacques Ouellet prolonge le bon coup d'envoi qu'il avait proposé avec son recueil Qui ose regarder ${ }^{12}$, lequel avait mérité, en 1987, cette récompense québécoise. Il s'agit ici, non seulement d'une continuité interrogative existentielle instaurée par les deux titres, mais encore d'un solide approfondissement de la pensée 
et du poème, de *la pensée du poème*. Sur un mode mineur, sans parures ni effets bruyants, le poète continue d'exprimer dans le détachement lent de la parole, cette distance prise en regard des opacités du réel: Tout s'en va le vent déplace / la chaise où j'écris / la lumière passe sur la feuille./ les signes se suivent / improbables subitement érigés / au milieu de la table nous formons / une tache mouvante dans le soir (p. 21). Il excelle encore à la saisie des heures oisives, à rendre cette rêverie de la passivité, cette attention distraite par le remuement tranquille du monde.

Ici, rien ne se passe (mais tout passe) dans le paisible défilé du poème qui dit l'inertie des choses et des paysages, dans les mots murmurés, effleurés: On' entend souvent n'importe où / une mer un lac une montagne / on peut entendre la chambre vide / mais c'est toujours soi / à son insu toujours (p. 16). Ce ton et cette mesure qui caractérisent déjà la poésie de Jacques Ouellet pourraient l'inscrire dans le prestigieux sillage d'un Jacques Brault, pour ces grandes atmosphères mélancoliques déployées dans l'exactitude littérale du poème, pour la capture radieuse des "moments fragiles"; on songe aussi pour le glissement de la diction, pour la pente sans appui de la pensée, à cette calme densité des poèmes de Marie Uguay. Et j'entends en ces correspondances, moins une gestion inexpérimentée des influences, que l'intuition d'* affinités électives ", l'apparentement à un lignage de la nostalgie et de l'émerveillement.

Car on trouve, chez ce poète, un sens tout à fait personnel de l'habitation méditative des lieux et du langage. On accède à des intérieurs d'où contempler les paysages: il y a des maisons, des chambres, des fenêtres, des vêtements, des objets dans cette poésie, tout cela suspendu dans le temps, dans l'espace, à l'instar des poèmes comme placés en suspens sur la page. Un livre donc, où perdurent longuement les échos de cette question sans réponse: où serons-nous dans une heure? (p. 39)...

Avec la ténuité attentive de la poésie de Jacques Ouellet, avec son * intuition de l'instant* intangible dans la quotidienneté du cérémonial amoureux - tu penches la tête / je ferme les yeux / c'est déjà le passé (p. 43) - contrastent le réalisme souvent cru voire le misérabilisme appuyé du texte d'André Paul, Devis des ruines neuves ${ }^{13}$. Ici, la ruine affective se dit dans la rancœur, en un langage qui ne parvient pas toujours à se délester du tumulte de la scène de ménage: Elle remue la viande / et chaque jour récite / les plus fortes aigreurs / de cet amour ancien / qu'elle a dû inventer. (p. 9) On sait bien que tout est dans le ton et que le mot *ordure", en poésie, n'est pas plus ordurier qu'un autre. C'est pourquoi, je veux moins insister, à propos de ce livre qui thématise jusqu'à l'obsession la ruelle crasseuse, la colique et l'engrais, l'assiette vide, l'attente de la paie et les débris de pneus, et où soupeser la bière rime avec claquer les portières (p. 31), 
sur la complaisance sans doute exacerbée du petit malheur qui devient grand que sur l'authenticité de telle saillie bien proche de l'indicible désarroi: Nous nous frôlons obstinément / vois-tu / l'un à l'autre invisibles. (p. 100) Dans le fatras des platitudes domestiques (Il suffisait alors de te concentrer / sur la précision du jet d'urine / sur l'inclinaison de la boîte de céréales / sur le son de l'eau dans la bouilloire [p. 58]), dans la révolte à l'égard de toute cette Conciergerie $d u$ tumulte (p. 29) qui exacerbe le désir amoureux, on entend parfois, avec la timidité des mots pour le dire, l'inconsolable rêve, à la manière parfois heurtée d'un Michel Beaulieu, de la tendresse: Pourtant tu entres ici en chantonnant. / Tu attardes tes paumes / sur son dos fatigué. / Tu es assez naïf pour quêter la tendresse / au milieu des cires, des solvants / et des lames. (p. 102) Malgré l'inflation métaphorique (les sacs de pudeur [p. 33] et le parapluie des confidences [p. 37]), malgré l'exaltation du dépit, du mal de vivre et du mal (d')écrire, je n'ai pas pu échapper, justement; au malaise de ce livre. Comme si la douleur pouvait se situer au-delà du poème: Je n'ai pas trouvé comment être deux et vivants. Ne m'attends pas. (p. 72)

Ambiguïté donc de la critique qui prétendrait prendre distance à l'égard de la poésie? Au fur et à mesure que je lis les recueils de poèmes, au fur et à mesure que j'écris ces lignes, je doute, bien sûr. Et plus encore devant les livres qui me paraissent divisés entre la sincérité de leurs propos et l'inadéquation des mots, comme impropres à traduire toute la vérité. En cette tension, pourtant, la poésie résiste ; comme s'il suffisait de quelques vers - prédestinés? - dans le magma d'un livre...

Ainsi, cet inconfort du poétique aux prises avec le scatologique, dans le livre d'André Paul, persiste avec les Blues en je mineur ${ }^{14}$ de Guy Marchamps: parle noir dans la nuit / et le jour se tiendra peinard / dans les chiottes du soleil (p. 38). Cette exhortation, comme tant d'autres dérisoires énoncés (Je commande trois poèmes "alldressed " pour me donner mal au cour [p. 36] et le monde est fou I comme de la bouse de vache [p. 65]), côtoient des vers d'une limpidité concise, fragments de miroirs égarés parmi les immondices: Je ne vois pas ce que tu vois / nos visions s'aveuglent / c'est dans le noir - que je te rejoins (p. 48). Nombre de scories, de fallacieux aphorismes ou de faciles jeux de mots se trouvent raturés par le déploiement ludique d'un long poème oral aux accents giguériens (Il y a des mots pour tout [p. 15 et ss.]: peut-être en est-il quelques-uns... de trop!), par l'harmonie d'une strophe à la découpe lumineuse: Tout ce que j'ai rêvé pour ces arbres majestueux / de chaque côté de la rue / cette lumière sur les feuilles / la brique rouge et l'asphalte / ces nuages qui nous prolongent dans le ciel. (p. 60) Il faut sans doute reconnaître aux blues de Guy Marchamps cette part de jubilante improvisation, cette spontanéité inhérente à l'atmosphère où ils se déploient. Mais reste, 
en poésie, le travail qui canalise l'euphorie. Ou encore, la sage consigne du silence: il y a la parole qui se vit / cherchant un sens que prendrait / la couleur du silence (p. 77).

Faudrait-il alors élever la voix, parler le haut et « beau * langage? Les poèmes de Joël Des Rosiers, dans Tribu ${ }^{15}$, ne résistent pas, quant à eux, à l'emphase d'une cour d'amour souvent empesée dans les factices élégances du passé simple (las..., nous quittâmes et nous dormîmes me laissent parfaitement éveillé), dans les vertiges de l'exclamation, les belles manières verbales, l'exotisme paré du mot rare et du néologisme. Mais, "La beauté sera moderne», rappelle la première partie du recueil; ainsi, l'obsolète amplitude du tón (ce goût pour ce qui est *dolent*, pour la * psalmodie *,...) voudra s'atténuer par la nomination des jeans (ils sont allégoriques [p. 21] ou assortis d'un coùrt smoking d'agneau [p. 29]: très excitant!), du sida, du blouson de cuir, du vidéoclip et du highway... En ce contrat mixte, indécis entre grand siècle et fin de siècle, se profilent des lectures. Mallarmé, peut-être, ou plutôt les Parnassiens: Le souvenir de vous m'étreint sur des images glacées / qui me furent offertes, où flâne un vaste effroi. (p. 19) Saint-John Perse, qui sait?: encore à l'arrimage aux môles de sable / ah lèvres jaillies de la géodésie des siècles / dites à elle que je suis las / de la jactance de l'amant (p. 15). Ou Rina Lasnier : la mort en sa fiction de malemort racheta / l'amour sur le tranchant de tes lèvres (p. 51). À moins que ne s'immiscent ici tels échos des lettres de Madame de Sévigné à sa fille de Grignan: Telle est la volupté de voir, indissociable de la / douleur de n'être pas vu. (p. 19)...

Je voudrais pourtant de l'admiration franche sous le couvert de cette ironie intertextuelle. Car on trouve, chez Joël Des Rosiers, par delà le hiératisme poseur, une telle passion d'écrire, un tel goût pour les subtilités de la langue, pour la phrase en équilibre, que l'exaltation même en vient à créer l'enchantement: l'air froid plein de tes longs pas / te voilà calme hors du simulacre / quelques jalousies levées sur de fraîches ténèbres / corps de lumière / éperdu dans le fiel du monde / la grâce va dedans les rues inciviles (p. 21). Efficace suite des monosyllabes en ce premier vers; ordre rythmique bien calculé de cette strophe portée par l'élan de la marche. Et quand la simplicité du naturel se substitue aux offices du culturel, l'émotion de la langue convie au recueillement: dans la langue de la mère / à l'abandon parmi les ruines langue / qui recueillit la charade de l'enfance / je songe à vous que je ne parle plus (p. 52).

Enfin, c'est peut-être dans les pages de prose (et ainsi, dans toute la troisième partie du livre, intitulée "Désir de désert") que Joël Des Rosiers réalise au mieux ses dons de poésie. Ce qui tient parfois de l'apprêt dans le poème devient alors foisonnement et luxuriance. Dans ses scènes marocaines qui relèvent du journal de voyage ou du reportage poétique, l'auteur rejoint, à l'occasion, la clairvoyance du 
Gide des Nourritures terrestres. Sens de la notation juste et de la description synesthésique, modulation de la syntaxe, judicieux effets de la ponctuation: Toute lumière désormais rougeoie sur les hommes, les bêtes, les kasbahs... et les femmes accroupies à l'ombre avare des murs en pisé dans leurs djellabas noires ne sont pas des chimères. (p. 95) Belle manière de tracer, en une longue phrase, la longueur d'une ombre et l'indécision d'un clair-obscur; d'exprimer, dans le suspense des points, l'écart spatial et la différence des sexes.

On sait bien que le temps modifie l'angle des lectures et que, par conséquent, le juste critique devrait toujours reprendre les siennes selon, à tout le moins, un plan quinquennal... Ainsi ce qui me paraît maintenant relever d'une certaine onction compassée, encombrant à l'occasion la vision de Joël Des Rosiers, pourra-t-il s'entendre, lors d'une lecture rendue moins myope par le recul temporel, comme un travail cumulatif de la poésie, comme une solidarité à l'égard de la longueur d'onde historique des textes ? Et ce qui semble aujourd'hui trivial parfois chez André Paul et chez Guy Marchamps tiendra-t-il demain du franc parler ou, par effet de rebours, d'une rabelaisienne humeur?

Ainsi, relisant les Textes 1976-1979 que Jean-Yves Collette reprend dans ses Propositions ${ }^{16}$, je me souviens que l'époque était, croyait-on, au textuel, au sexuel, au corps-texte, à l'euphorique dissection des formes dans l'asepsie des salles de chirurgie... Pourtant, juste retour des choses, ce qui me retient maintenant dans la poésie de Collette, c'est bien plutôt la fougue lyrique de l'érotisme, la spontanéité des scènes saisies, cette gourmandise du poème devant ce qu'il voit et donne à voir: le théâtre de ta jupe retroussée / et maquillée au tournant d'une rue / ton corps senti comme une indiscrétion / jusqu'au ruissellement habituel / dans les herbages (p. 19). Ici, la reprise du poème en version révisée, sa constante répétition générale de lui-même constitue une forme de résistance à la fragmentation, une volonté de parfaire les textes par le travail, la rature, la "proposition " poétique renouvelée; la ré-écriture, alors, vise moins au déni de l'état premier du texte qu'à une tentative de le doubler, de le redoubler, comme pour en sonder l'innombrable variété des possibles.

La poésie s'inscrit alors comme rituel, comme rythme verbal sans cesse soumis à l'expérience de l'atelier, véritable * work in progress ". Et si cet exercice de l'épure s'interdit le poétisme sentimental ou la métaphore embellie, il parvient à rendre, dans l'impeccable dépouillement d'une syntaxe aux simples subtilités, dans le sens du souffle bien coupé, toute la turbulence de la passion et le vertige grave des appétits: Une rare nuance apportée, des volées de feu traversant le ciel et la vitesse du vent, dont la chair n'a pas l'habitude, s'insinue entre les doigts noués. La lourde couleur et l'influence des femmes, jouant du corps désespéré, et l'intervention de la mort dans la tête pliée. (p. 102) 
Chez Jean-Yves Collette, le texte amoureux se trame encore dans la nomenclature des jouissances où s'exaltent le sang, le sperme, la salive et toutes nourritures; écrire dans la saveur émerveillée du corps et du poème - tel un blason du corps: tes longs et beaux ruisseaux / ou bien tes épaules blanches / ou bien cette bouche entre tes jambes / qui sent les fruits / et où l'on entre / pénétrant dans une ville pavoisée (p. 21). Ces états torrides se trouvent assortis d'une sensualité parfois plus moelleuse où dans l'ironique rigueur de la métrique, se donnera à entendre - nostalgie encore... - telle nelliganienne musique: je t'ai 'vue longtemps tous les jours amoureuse / caresser distraitement le rebord d'un verre / et mouiller ton doigt dans le vin vert / le lécher ensuite une minute délicieuse (p. 48).

Chez Jean-Yves Collette, la poésie n'est toujours qu'une hypothèse provisoire d'elle-même; à concilier le collage, la citation ou le fragment, elle est tout autant une expérience de langage et d'émotion qu'un attentif exercice de style. Au plaisir de retrouver ces textes s'ajoute encore celui de participer à un labeur où $[l] a$ forme [...] est à la fois lyrique et contrainte; le lyrisme y est contraint par la rigueur, par l'essentiel, par le désespéré. (p. 121)

Étranges coïncidences entre les textes, énigmatique circulation osmotique des faits de poésie. Ainsi je lis, dans le recueil de Michel R. Guay, l'Inexpiable suivi de Textes baroques et autres excès ${ }^{17}$, cette «proposition * qu'un jeu d'attribution poétique m'aurait peut-être fait allouer à Jean-Yves Collette: L'articulation des propositions: / laisser voir qu'on s'émerveille / sans mourir de ce deuil saisissant / ou vouloir dire fini le temps, la cécité / de répondre (p. 14). Pourtant, en dépit de la facture fortement articulée de nombre des poèmes de Guay, la radieuse excitation textuelle caractéristique de la poésie de Collette fait place ici à un certain laconisme de la pensée. Fréquence de l'infinitif, ce temps mort, obsession de l'accès (entrer c'est mourir [p. 20]), du seuil (Invisible seuil [p. 25]) et du faux pas (Cela fait faux pas [(p. 26]), assomption de l'absence et de la vacuité (Le vide - la tête d'idées - / condition préalable à tout portrait intérieur [p. 44]): plusieurs traits sémantiques et formels concourent donc à instaurer une distance entre le lecteur et le poème, comme si la poésie même résistait au plaisir, au désir. Tentation d'exister, attentes et frissons, / désir sans résistance écaillé (p. 53). Pareil étalement substantif induit un texte monochrome, une langue étale sans grande secousse ni surprise. Rien de moins "baroque " ou d'" excessif", nulle exubérance débridée, me semble-t-il, dans ce livre tenu par la mallarméenne ambition de * faire le vide ", d'assumer le blanc, et où "ça parle ", pardelà un immense silence, dans une sorte de monotonie récitative, en un déroulement de langage qui ne parvient pas à heurter l'oreille: L'agitation la même / sentiment / d'une machination / comme d'agir (p. 80). Avouons que j'ai du mal avec le poème qui tente d'instruire ou 
de corriger (Présage de bassesse. / Doses bibliques. / Nos / queues. [p. 64]), avec la transgression sans choc ou la lourdeur de l'exposé: Exposé à l'insoutenable prospérité / des contemplatifs, j'écartai d'instinct / mes graffiti simiesques. (p. 30) Me ravit plutôt cet imperceptible fondu entre les mots et les choses, cette indétermination entre le langage et le regard qu'il porte sur le monde: Pêchais à la ligne / que le lac, / lucidité hagarde, labile, / château d'eau s'abolissant, / tari. (p. 47) Qui mesure la distance du plus simple à l'essentiel, de l'apparente facilité (fragilité) à la profondeur à laquelle ce livre atteint - quand il délaisse la * démonstration * pour la * proposition *?

Si le portrait intérieur (p. 44) auquel nous convie le livre de Michel R. Guay ne réussit pas toujours à se tracer faute d'une incarnation viscérale du sujet dans la pulpe du langage, si ce corps qui s'expose a du mal à téter des cailloux (p. 89), rien de tel dans le livre d'André Gervais, La nuit se lève ${ }^{18}$, véritable machine magique où toute matière littérale se trouve broyée, pulvérisée, emportée par l'énergie passionnée de l'explosion et de la trituration. Incidence de la moindre capillarité textuelle, lettrisme ludique, compétence étymologique et circulation translinguistique, tous les recours. méthodologiques de ce texte traduisent la langue en procès: De là l'être à la lettre, jusqu'à l'incendie. (p. 9)

Cette formule d'André Gervais pourrait bien nous permettre une mise en relation thématique avec les livres d'Anne-Marie Alonzo et d'Hélène Dorion; mais le lecteur (l'interlocuteur...) familier des manœuvres linguistiques du premier percevra d'emblée ici la préséance formelle de l'unité alphabétique sur la confidence épistolaire... Encore que supporte cette comparaison, tel aveu plus intimiste: avoir cours et course d'amour mais, pendant le deuil, être, sans autre forme de procès, délavé de ratures (p. 20). Qu'on ne s'y méprenne pas; je ne vais pas, histoire d'accroître la coïncidence entre les recueils ici commentés et qui tiennent, en diverses stratégies de fragmentation, discours d'amour ou de deuils amoureux, inscrire Gervais dans le lignage de la poésie élégiaque ou courtoise... Même si, persistent ici comme ailleurs, des séquelles de la blessure: pour quel inappropriable été blessé / de ta chair inlassablement ouverte / pour le vertige de la mienne (p. 118).

Ce qui subjugue voire ce qui envoûte, chez Gervais, tient d'abord dans l'énergie effrénée de la diction, dans l'ardeur d'une "désautomatisation » systématique du discours. Il ne s'agit pas du seul bonheur - comme en enfance retrouvée/retournée - de jouer avec les mots; il faut illustrer l'évidence matérielle du langage, infiniment malléable et si souvent " manipulé * par ce que d'aucuns appellent " communication *. Pour ce poète, la * facture " réside, forcément, dans la fracture (p. 84), celle qui retient l'attention. Comme, en musique, la syncope ou le suspense: la robe havarde a capella d'odeurs / dans 
le buisson et l'envergure incorrigée [...] la robe havarde a capella d'odeurs / tranche le signet du lecteur qui (p. 114).

Altruiste et pudique à la fois, l'écriture de Gervais maintient le sujet à distance. « Je " ne parle et ne se désigne que dans son effort d'évidence toujours voué à l'échec - de présentation à l'autre: étant d'instance à ne pas savoir si je parle de moi dans les lumières du sujet (p. 69). Qui parle? Telle est la question incessamment répétée: $Q u i$ parle, ravi par le dispositif négatif, en quelque sorte. C'est la question. De la présentation, de l'autre, en tant qu'autoportrait. (p. 125) Le portrait comme l'«autoportrait * ne peuvent s'interroger ailleurs que dans la scène énigmatique du miroir: De l'autre, en tant qu'autoportrait (p. 64); tant il est vrai que il faut changer de nom tout le temps si on ne veut pas se laisser constater (p. 25). Cette effervescence de la métamorphose identitaire enjoint le langage à se déployer dans sa différence et son "étrangeté *. Le texte, dès lors, n'a pas d'autre enjeu que celui de la démarcation, de l'écart, de la subversion en regard des conventions poétiques. Dans la dissémination et l'essaimage, dans la perturbation de tous les codes, il se propose comme kaléidoscope verbal, lieu d'une unité émiettée, centre clos de l'éparpillement du sujet, du discours: dans cette incidhente il décline / son identité d'être épars / de la coquetterie à l'anecdote / avant de disparaître et d'en découdre / du fil de l'eau et de la conversation (p. 116). Abolition de l'ordre langagier, bruissement perpétuel du sens trituré, démis de ses usages courants, là résident donc le projet et le travail de cette poésie. État de choc des mots et des choses.

Mais la virulence fragmentaire n'exclut pas ici le moment lisible, le verbe ou le vers d'« état ": mais l'arbre est dans / ses feuilles et le drap est dans / sa voix (p. 68). La volubilité de la voix qui se brise contre le langage connaît la lucidité de sa perte et de son ravissement: [...] et pourtant on détient des épures, et pourtant on retient des formes, on écrit alors, dejà, perdu, saisi (p. 19). J'admire encore, chez André Gervais, cette exigence sans concession à l'égard du livre: structuration dynamique et contrepoint dense entre les fragments, forte cohérence de l'appareillage paratextuel, investissement réflexif et conceptuel au sein de son propre laboratoire de langage. Et s'il y a de l'utopie - celle de redécouvrir Babel, celle de parler toutes les langues en une seule et d'inscrire tous les textes en un Texte: comme en ce Pendule de Foucault - dans la patience de ce travail vertigineusement spéculaire, elle sollicite la solidarité des efforts. Cette écriture sans compromis réclame une lecture sans distraction: En quoi se désigne ici d'un mot le travail singulier de l'un ("salut G. ") et, par une exacte inversion, de l'autre ("j'ai lu ça»). (p. 138) Salut G.!

1 Hubert Aquin, Prochain Episode, Montréal, le Cercle du livre de France, 1978, p. 97. 
2 Claude Beausoleil, Une certaine fin de siècle, Saint-Lambert, Editions du Noroit, 1983, p. 114.

3 Anne-Marie Alonzo, l'Immobile, Montreal, l'Hexagone, 1990, $150 \mathrm{p}$.

4 Id., Geste, Paris, Editions des Femmes, 1979, $147 \mathrm{p}$.

5 Id., Veille, Paris, Editions des Femmes, 1982, 99 p.

6 Hélène Dorion, Un visage appuyé contre le monde, Saint-Lambert, Éditions du Noroit et Chaillé-sous-les-Ormeaux, Dé Bleu, 1990, 112 p. (avec quatre dessins de Marc Garneau).

7 Jean Charlebois, Confidentielles, Saint-Lambert, Éditions du Noroit, 1990, 122 p. (avec une huile et dix dessins de Marc-Antoine Nadeau).

8 Id., Hanches neige, Saint-Lambert, Éditions du Noroft, 1977, [n.p.].

9 Id., Corps cible, Saint-Lambert, Éditions du Noroit, 1988, $128 \mathrm{p}$.

10 André Marquis, Nul vieux château, Montréal, les Herbes rouges, 1990, 100 p.

11 Jacques Ouellet, Qui ose regarder, Montréal, Leméac, 1987, 60 p.

12 Id., Où serons-nous dans une heure, Saint-Lambert, Editions du Noroft, 1990, 78 p. (avec trois eaux-fortes de Nicole G.-Ouellet).

13 André Paul, Devis des ruines neuves, Saint-Lambert, Editions du Noroft, 1990, 120 p. (avec huit dessins de Yvonne Lammerich).

14 Guy Marchamps, Blues en je mineur, Saint-Lambert, Editions du Noroit, 1990, $80 \mathrm{p}$. (avec six dessins de Yves Cadorette).

15 Joel Des Rosiers, Tribu, Montreal, Triptyque, 1990, $112 \mathrm{p}$.

16 Jean-Yves Collette, Propositions, Textes 1976-1979, Saint-Lambert, Editions du Noroft, 1990, $176 \mathrm{p}$.

17 Michel R. Guay, l'Inexpiable suivi de Textes baroques et autres excès, SaintLambert, Éditions du Noroft, 1990, 96 p. (avec cinq encres de France Lachaine).

18 Andre Gervais, La nuit se lève, Saint-Lambert, Editions du Noroft, 1990, 176 p. (avec un tableau de Bruno Santerre). 